\title{
DEP et flux de données
}

\section{Yvonne Gilli}

Dr méd., membre du Comité central de la FMH, Département Numérisation / eHealth

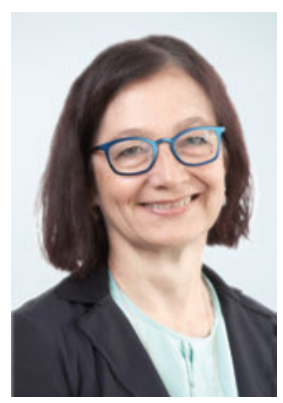

L'expérience provocatrice du psychologue Stanley Milgram sur la soumission à l'autorité (un sujet inflige des chocs électriques de plus en plus forts à un élève pour le punir) a marqué l'histoire. Cette expérience traumatisante a suscité la controverse auprès de la communauté scientifique et, aujourd'hui, elle ne s'explique que par la volonté historique de travail de mémoire en lien avec les crimes du nazisme. Plus tard, Milgram a lui-même pris ses distances par rapport à cette étude socio-psychologique. Parmi ses travaux, d'autres sont moins connus, comme son hypothèse formulée dès les années 70: la "Urban Overload Hypothesis», selon laquelle les citadins changent leur comportement social en raison de la surcharge sensorielle. Peut-être qu'en cette période de surabondance d'informations, il est grand temps de poser un regard nouveau sur cette hypothèse. Indépendamment des données de santé produites par les objets connectés, le volume des données numériques consommées par un hôpital oscillent aujourd'hui au niveau du pétaoctet $\left(10^{15}\right.$ octets).

\section{Les hôpitaux inscriront davantage de données} dans le DEP que dans le rapport de sortie habituel.

Une étude du Veterans Affairs Medical Center de 2010 a établi un lien entre le fait de ne pas voir un message et une surabondance d'informations dans les dossiers médicaux informatisés. Avec le dossier électronique du patient (DEP), le corps médical disposera d'une source supplémentaire d'informations électroniques. L'avenir se profile déjà, les hôpitaux inscriront davantage de données sur les patients dans le DEP que dans le rapport de sortie habituel. Cette évolution est l'expression d'une mutation numérique selon laquelle l'interprétation d'un résultat clinique ou aussi une valeur isolée peuvent être rapidement agrégées, cumulées et transportées.

Le journaliste américain Clay Shirky a trouvé un dénominateur commun pour le problème du flux accru d'informations: «It's not information overload. It's filter failure.» Ce paradigme fonctionne dans le monde des médias sociaux et le filtre anti-spam de ma boîte de réception accomplit également un travail utile. En revanche, il semble encore inimaginable, du moins dans un futur proche, de réduire dans le DEP le nombre croissant et la complexité des données nécessaires au traitement.

La Loi sur le dossier électronique du patient (LDEP) et ses ordonnances ne disent rien de concret sur quelles données doivent être saisies dans le DEP ni ne répondent à la question de savoir dans quelle mesure le corps médical est tenu d'utiliser le DEP comme source d'information. Renoncer volontairement à ces précisions concrètes oblige les médecins qui veulent se raccorder au DEP à relever le défi de remplir la fonction du DEP tout en satisfaisant aux dispositions légales de la protection des données et du secret médical, sans oublier la menace des sanctions prévues par le Code pénal. Tout ceci est une question de devoir de diligence qui n'est pas - et c'est tout à fait compréhensible - réglée dans la LDEP, mais qui doit quand même être évaluée au cas par cas. Une erreur sera facile à prouver car toutes les saisies et toutes les modifications de données sont enregistrées dans le DEP.

Pour les médecins raccordés volontairement à l'infrastructure du DEP, l'absence de dispositions concrètes sur la manière de gérer le DEP constitue pour l'instant un risque en matière de responsabilité dont la portée n'est pas encore connue. Ces insécurités juridiques pourraient inciter une partie importante du corps médical, soit à renoncer à se raccorder à l'infrastructure

\section{Les insécurités juridiques pourraient inciter une partie du corps médical à renoncer à se raccorder à l'infrastructure du DEP.}

du DEP ou, inversement, à inscrire toutes les données importantes dans le DEP, sans qu'on puisse pronostiquer avec certitude la pertinence future des données au moment de leur saisie. Ces deux manières de procéder sont certes motivées par des risques liés à des questions de responsabilité mais elles empêchent une communication efficace entre professionnels de santé. 